\title{
Visual Hallucinations Secondary to Infarction of the Caudate
}

Pouya Entezami ${ }^{1}$, Marika Raff ${ }^{2}$ and Sarah Bonner ${ }^{*}$

${ }^{1}$ University of Toledo College of Medicine, Toledo, USA

${ }^{2}$ University of Michigan Medical School, Ann Arbor, USA

${ }^{3}$ St. Joseph Mercy Health System, Ann Arbor, USA

*Corresponding author: Sarah M Bonner, IHA Hospital Medicine Service, 24 Frank Lloyd Wright Drive, PO Box 0446 Lobby J, Ann Arbor MI 48106 , USA, Tel: 877.336.6307; Fax: 734.712.3855; E-mail: Pouya.Entezami@rockets.utoledo.edu

Received date: May 30, 2015; Accepted date: June 22, 2015; Published date: June 27, 2015

Copyright: $\odot 2015$ Entezami $P$, et al. This is an open-access article distributed under the terms of the Creative Commons Attribution License, which permits unrestricted use, distribution, and reproduction in any medium, provided the original author and source are credited.

\section{To the editor}

A 40-year-old male presented with a three day history of dizziness, gait disturbance, and worsening visual hallucinations consisting of fire and person imagery. History included two prior cerebral infarctions in 2010 and 2011 involving the left caudate, cerebellum, and lentiform nucleus from which he developed persistent visual hallucinations that were intermittently exacerbated by unknown causes. On exam, his cognition was mildly impaired, though the remainder of the neurological exam was unremarkable. The patient also described periods of confusion that worsen with fatigue.

On admission, the patient was alert and oriented. Language function was normal including naming, repetition, comprehension and fluency. Attention and concentration were intact while fund of knowledge was slightly impaired and cognition was mildly slowed. There were no sensory, motor, or visual deficits, and coordination was intact. Magnetic resonance imaging demonstrated no acute areas of ischemia or hemorrhage (Figure 1), but did show ventricular dilatation consistent with the location of his prior strokes (Figure 2); diffusionweighted imaging (DWI) was negative for acute changes as well. The dizziness and gait disturbance resolved within three days of admission and the hallucinations, attributed to the prior left caudate stroke, returned to baseline.

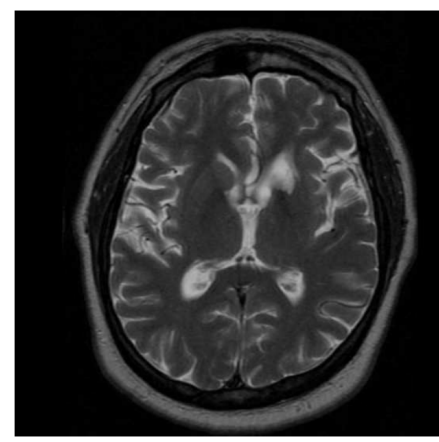

Figure 1: T2-weighted MRI image done on admission demonstrating no new acute intracranial pathology

Several neuropsychiatric pathologies secondary to strokes have previously been described, including depression, anxiety, catastrophic reactions, and rarely psychosis. Rabins et al. described five patients who developed Schizophreniform psychosis due to frontoparietal strokes with varying degrees of hallucination $[1,2]$. Similarly, a few cases of psychosis following thalamic [3-6] and putaminal [7] infarctions have been reported. Caudate nucleus lesions leading to psychosis and/or hallucinations have also been uncommonly described. Supplied by the lenticulostriate artery, the caudate nucleus modulates motor function and several cognitive functions, including memory, emotion, and language $[5,6,8,9]$. Thus, a vascular event implicating the lenticulostriate artery causing infarction of the caudate is a rare but possible etiology for visual hallucinations, and while exceedingly rare, thalamic or basal ganglia infarction should be considered when reviewing imaging for new-onset cases of psychosis. It is important to note that for older patients with similar symptoms, dementia with Lewy Bodies should be strongly considered as a differential diagnosis.

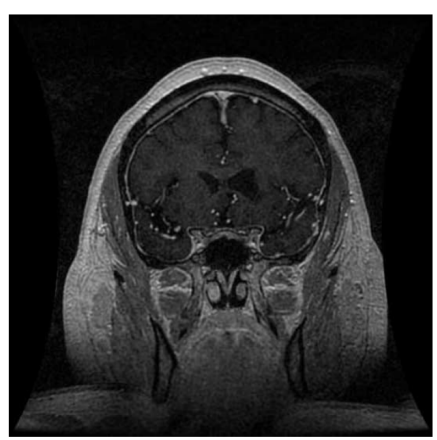

Figure 2: Post-contrast T1-weighted MRI image demonstrating leftventricular dilatation due to atrophy, suggestive of past basal ganglia stroke.

\section{References}

1. Chemerinski E, Robinson RG (2000) The neuropsychiatry of stroke. Psychosomatics 41: 5-14.

2. Rabins PV, Starkstein SE, Robinson RG (1991) Risk factors for developing atypical (schizophreniform) psychosis following stroke. J Neuropsychiatry Clin Neurosci 3: 6-9.

3. Serra Catafau J, Rubio F, Peres Serra J (1992) Peduncular hallucinosis associated with posterior thalamic infarction. J Neurol 239: 89-90.

4. McGilchrist I, Goldstein LH, Jadresic D, Fenwick P (1993) Thalamofrontal psychosis. Br J Psychiatry 163: 113-115.

5. Santos S, Alberti O, Corbalán T, Cortina MT (2009) [Stroke-psychosis. Description of two cases]. Actas Esp Psiquiatr 37: 240-242.

6. Lee S, Kim DY, Kim JS, Manda S, Danilov L, et al. (2010) When Should Brain Imaging be Performed?: A Case Report of Caudate Nucleus Infarct. Psychiatry (Edgmont) 7: 31-33. 
Citation: Entezami P, Raff M, Bonner S (2015) Visual Hallucinations Secondary to Infarction of the Caudate. J Neurol Neurophysiol 6: 298. doi: 10.4172/2155-9562.1000298

Page 2 of 2

7. Kitabayashi Y, Narumoto J, Otakara C, Hyungin C, Fukui K, et al. (2006) Schizophrenia-like psychosis following right putaminal infarction. J Neuropsychiatry Clin Neurosci 18: 561-562.

8. Lee S, Kim DY, Kim JS, Kaur G, Lippmann S (2011) Visual hallucinations following a left-sided unilateral tuberothalamic artery infarction. Innov Clin Neurosci 8: 31-34.
9. $\quad$ Kaur R (2012). "Post Stroke Psychosis." Delhi Psychiatric Journal 15: $221-222$ 